APS/123-QED

\title{
Long-lived electron spin coherence in CdSe/ZnSSe self-assembled quantum dots
}

\author{
M. Syperek, ${ }^{1,2}$ D. R. Yakovlev, ${ }^{1,3}$ I. A. Yugova,,${ }^{1,4}$ J. Misiewicz, ${ }^{2}$ I. V. \\ Sedova, ${ }^{3}$ S. V. Sorokin, ${ }^{3}$ A. A. Toropov, ${ }^{3}$ S. V. Ivanov, ${ }^{3}$ and M. Bayer ${ }^{1}$ \\ ${ }^{1}$ Experimentelle Physik 2, Technische Universität Dortmund, 44221 Dortmund, Germany \\ ${ }^{2}$ Institute of Physics, Wroctaw University of Technology, 50-370 Wroctaw, Poland \\ ${ }^{3}$ Ioffe Physical-Technical Institute, Russian Academy of Sciences, 194021 St.Petersburg, Russia \\ ${ }^{4}$ Physical Faculty of St.Petersburg State University, 198504 St. Petersburg, Russia
}

(Dated: February 12, 2018)

\begin{abstract}
The electron spin coherence in $n$-doped and undoped, self-assembled $\mathrm{CdSe} / \mathrm{Zn}(\mathrm{S}, \mathrm{Se})$ quantum dots has been studied by time-resolved pump-probe Kerr rotation. Long-lived spin coherence persisting up to $13 \mathrm{~ns}$ after spin orientation has been found in the $n$-doped quantum dots, outlasting significantly the lifetimes of charge neutral and negatively charged excitons of $350-530$ ps. The electron spin dephasing time as long as $5.6 \mathrm{~ns}$ has been measured in a magnetic field of $0.25 \mathrm{~T}$. Hyperfine interaction of resident electrons with a nuclear spin fluctuations is suggested as the main limiting factor for the dephasing time. The efficiency of this mechanism in II-VI and III-V quantum dots is analyzed.
\end{abstract}

PACS numbers: 78.66.Hf, 78.47.jd, 78.47.jh, 75.75.-c, 78.67.Hc, 78.47.-p

\section{INTRODUCTION}

The electron spin coherence in semiconductor quantum dots (QDs) has been extensively studied experimentally and theoretically in recent years. [1 3] This activity is driven by prospective applications of the electron spin in quantum information processing requiring methods of initialization, manipulation, storage, and readout of spin coherence. A vital problem in this respect concerns the coupling of the electron spin to its environment, deteriorating the spin dynamics. The strength of this interaction determines the transverse spin relaxation time $T_{2}$, limiting coherent state manipulation. In QDs the electron wave function is strongly spatially localized by the three-dimensional confinement potential. On one hand this leads to discrete energy levels and makes the electron spin weakly susceptible to the main spin relaxation mechanisms in bulk semiconductors related to the spin-orbit interaction. 4] On the other hand it enhances the Fermi contact hyperfine interaction, which therefore is considered as main relaxation mechanism for QD electron spins. [5, 6] The hyperfine interaction is controlled by: (1) the nuclear isotope composition, (2) the hyperfine coupling constants, (ii) the number of lattice nuclei, and (iv) the nuclear spin magnitudes. Therefore, a proper choice of the QD semiconductor material may minimize this interaction, potentially prolonging the transverse spin relaxation time. In this respect II-VI semiconductors are an interesting alternative to III-V materials. For example, in CdSe the abundance of nuclear isotopes with nonzero spin is $7.6 \%$ for Se and $25 \%$ for Cd only, compared with the $100 \%$ for In, Ga and As in the widely studied (In, Ga)As dots. [7] Therefore, the electron spin dephasing by the hyperfine interaction should be considerably weaker in CdSe dots.

Coherent electron spin dynamics was studied by timeresolved Faraday rotation for colloidal CdSe QDs 8 and $\mathrm{Cd}(\mathrm{S}, \mathrm{Se}) \mathrm{QDs}$ in semiconductor-doped glasses [13], both of wurtzite-type. Longitudinal spin relaxation times of $T_{1} \sim 20 \mu \mathrm{s}$ and transverse spin dephasing times $T_{2}^{*} \sim 3$ ns were reported. [8, 9] The measured Faraday rotation traces show complicated quantum beat patterns due to the QD-anisotropy typical for wurtzite structures (leading to a complex exciton fine structure) and also due to the random dot orientation in the ensemble, so that the optical selection rules become undefined. These factors complicate the theoretical description of coherent spin dynamics in colloidal QDs, in addition to the influence of surface states. [9]

Some of these complications become resolved in epitaxially-grown self-assembled QDs. First, the influence of surface states is eliminated. Second, modulation doping allows one to fabricate charged QDs with a single resident electron per dot. 14] The spin dynamics of resident electrons is not limited by radiative exciton recombination as in empty QDs. Resident electron spin coherence can be generated via an intermediate trion state, as suggested theoretically 15 and demonstrated experimentally in (In,Ga)As/GaAs QDs. 14, 16] Another advantage of self-assembled QDs is their well-defined crystallographic orientation on the substrate, from which optical selection rules are established. Also the crystal structure can be controlled by the choice of substrate material, e.g., grown on a GaAs substrate, CdSe QDs have cubic symmetry instead of wurtzite symmetry, simplifying the exciton fine structure.

In this paper we report on time-resolved pump-probe Kerr rotation (KR) studies of the electron spin coherence in $n$-doped and undoped self-assembled $\mathrm{CdSe} / \mathrm{Zn}(\mathrm{S}, \mathrm{Se})$ QDs with a cubic crystal structure. We observe longlived spin beats related to the coherent precession of electron spins about a transverse magnetic field. The coherence persists over times exceeding the interval between subsequent laser pulses of $\sim 13$ ns. Analysis of the $\mathrm{KR}$ traces as function of magnetic field gives information about dephasing times and $g$ factors of the resident 
QD electrons. Also the temperature dependence of the dephasing time was investigated, and the results are compared to literature data, especially for colloidal wurtzite CdSe QDs.

\section{EXPERIMENT}

The investigated CdSe/Zn(S,Se) QD multilayer structures were pseudomorphically grown by molecular-beam epitaxy on a GaAs:Si buffer layer on top of an $n$-type GaAs (001) substrate. First, a $1.5 \mu$ m-thick $n$-type ZnMgSSe:Cl wide-gap confining layer $\left(E_{g}=2.96 \mathrm{eV}\right.$ at $T=77 \mathrm{~K}$ ) was deposited to separate the $\mathrm{QD}$ region from the GaAs buffer. Then, ten periods of CdSe QD layers (each having a nominal thickness of $2.5 \mathrm{ML}$ with a self-assembled dot density of $5-8 \times 10^{10} \mathrm{~cm}^{-2}$ ) were grown, each layer sandwiched between $\mathrm{Zn}(\mathrm{S}, \mathrm{Se}) / \mathrm{ZnSe}$ short-period superlattice (SL) barriers with a thickness of $50 \mathrm{~nm}$. The compressive strain induced by the CdSe QDs is compensated by the tensile strain intentionally generated in the SL barriers, which provides similar growth conditions for each QD layer. Finally, the entire structure was capped by a $30 \mathrm{~nm}$-thick ZnMgSSe layer. Two structures were fabricated: a reference sample with nominally undoped QDs and a sample with $n$-doped QDs. In the $n$-doped structure, the middle part of each SL barrier with a thickness of $17 \mathrm{~nm}$ was doped by $\mathrm{Cl}$ with a concentration resulting in an electron sheet density of $(3-5) \times 10^{11} \mathrm{~cm}^{-2}$. We expect that only $10-20 \%$ of the electrons are transferred to the QDs through the undoped $17 \mathrm{~nm}$-thick SL spacers. From these numbers we expect that the QD ensemble represents mostly a mix of charge-neutral and singly-charged QDs, but there may be also a small fraction of doubly or higher charged dots.

The pulsed laser source used in the experiments was an optical parametric oscillator pumped by a mode-locked Ti:Sapphire laser. The laser system generates pulses with a duration of $1.6 \mathrm{ps}$ at a repetition rate of $75.6 \mathrm{MHz}$, corresponding to $13.2 \mathrm{~ns}$ pulse separation. The photon energy could be tuned in the range $2.38-2.47 \mathrm{eV}$. The samples were held in a helium bath cryostat equipped with a superconducting split-coil magnet. Magnetic fields $\mathbf{B}$ up to $7 \mathrm{~T}$ were applied normal to the structure growth $z$-axis (Voigt geometry, $\mathbf{B} \perp \mathbf{z}$ ). The sample temperature was varied from 1.6 up to $50 \mathrm{~K}$.

In the KR experiment the laser beam was split into a circularly polarized pump and a linearly polarized probe. Both beams were focused on the sample with a spot diameter of $\sim 150 \mu \mathrm{m}$. To avoid dynamic nuclear polarization, the helicity of the pump beam was modulated at $50 \mathrm{kHz}$ rate using a photoelastic modulator. The pump pulse excites carriers with spins polarized along the $z$ axis. The subsequent coherent spin dynamics in form of Larmor precession about $\mathbf{B}$ is measured by the rotation angle $\Theta_{K R}$ of the polarization plane of the probe. To detect $\Theta_{K R}$, a homodyne technique based on phasesensitive balanced detection was used. 14]
The spectrally-resolved recombination dynamics of the optically excited electron-hole pairs could be detected in time-resolved photoluminescence (TRPL). For that purpose the samples were mounted in a helium flow cryostat at $T=8 \mathrm{~K}$. The PL emission was dispersed by a $0.5 \mathrm{~m}$ spectrometer and detected by a synchroscan streak camera with an S20 photocathode. The overall time resolution of this setup was 15 ps.

\section{RESULTS AND DISCUSSION}

The low temperature photoluminescence spectrum of the $n$-doped CdSe/Zn(S,Se) QDs under quasi-resonant excitation (see arrow indicating the laser photon energy) is shown in Fig. 1(a). The QD emission due to radiative recombination of negatively charged excitons (trions) in singly-charged dots and of charge neutral excitons in empty QDs is centered at $2.42 \mathrm{eV}$. The emission is characterized by considerable spectral broadening of $\sim 50 \mathrm{meV}$ due to QD inhomogeneities. A similar PL spectrum is obtained for the undoped QDs (not shown).

The exciton recombination dynamics was measured by
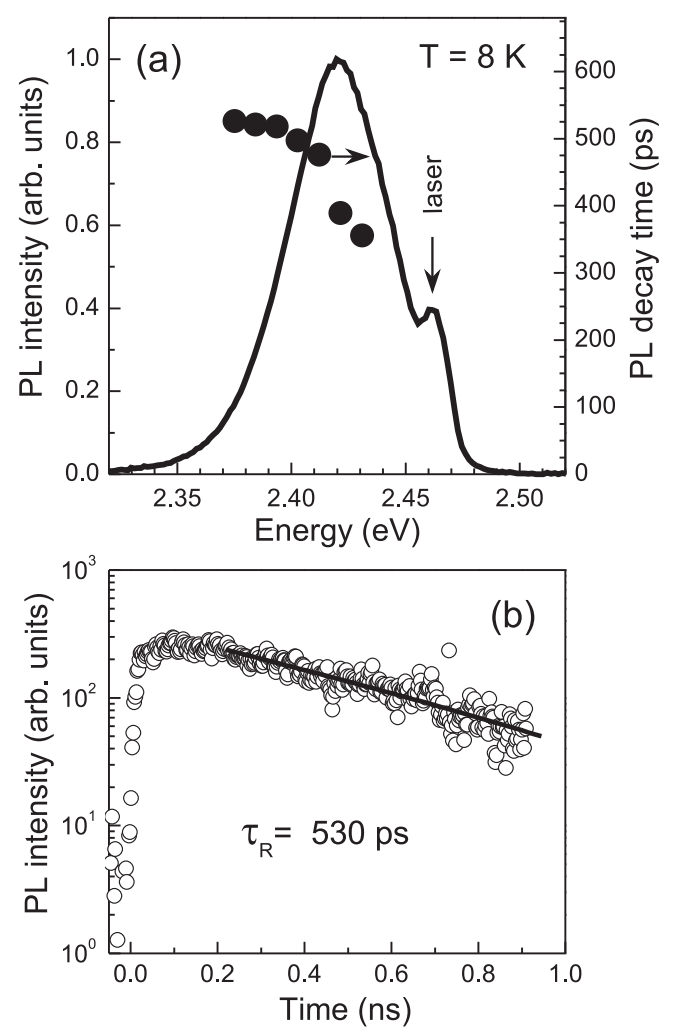

FIG. 1: (a) Photoluminescence spectrum (line) and photoluminescence decay time dispersion (circles) of the $n$-doped $\mathrm{CdSe} / \mathrm{Zn}(\mathrm{S}, \mathrm{Se}) \mathrm{QDs}$ measured for quasi-resonant excitation at $2.46 \mathrm{eV}$ photon energy. (b) Example of the PL intensity decay at $2.38 \mathrm{eV}$ after pulsed excitation, plotted on a logarithmic scale: circles - experiment, line - fit. 
TRPL. An example of the PL decay at $2.38 \mathrm{eV}$ is shown in Fig. T(b). The decay can be fitted well by a single exponential as shown by the solid line with a characteristic time of $\tau_{R}=530 \mathrm{ps}$. The PL decay time varies across the emission band: $\tau_{R}$ increases from $350 \mathrm{ps}$ on the high energy flank at $2.43 \mathrm{eV}$ to $530 \mathrm{ps}$ on the low energy side at $2.38 \mathrm{eV}$, see circles in Fig. 1(a). The observed systematic increase of the decay times with decreasing transition energy is in agreement with previous measurements on epitaxially-grown $\mathrm{CdSe} / \mathrm{ZnSe} \mathrm{QDs}$, both for ensembles [17 and single dots 18. The increase in the exciton radiative lifetime with increasing localization energy can be explained by a decrease of the exciton coherence volume in real space and, hence, its increased spread in K space 19]. We note here, that in the studied structures the effective barrier energy provided by the superlattice minibands is $2.84 \mathrm{eV}$, which implies the strong confinement regime for the confined electron and hole wavefunctions.

The spin dynamics was investigated by pump-probe Kerr rotation. Figure 2(a) shows the KR trace for the $n$-doped QDs measured at $B=0.5 \mathrm{~T}$. The laser photon energy was tuned to $2.44 \mathrm{eV}$ for resonant excitation of the trion and exciton states in the QDs. The KR signal shows features typical for structures containing singly-charged QDs or quantum wells with a low density electron gas. 14] At positive time delays relative to the pump pulse at time zero, a periodically oscillating signal with exponentially decreasing amplitude for increasing delays is observed. Remarkably the signal amplitude is still strong at delays of $4.5 \mathrm{~ns}$, i.e., at times strongly exceeding the characteristic trion lifetimes, that do not exceed $0.6 \mathrm{~ns}$, see Fig. 1(a). This allows us to conclude that the KR signal is dominated by the contribution of the resident electrons in singly-charged QDs, whose lifetime is not limited by recombination. A closer look at negative time delays, see the red line in Fig. 2(a), reveals spin beats with rather small amplitude.

The spin precession frequencies can be evaluated by Fast Fourier Transformation (FFT) as shown in Figs. 2(b) and 2(c). The precession frequencies for negative and positive delays are close to each other: $\omega_{L}=8 \pm 1 \mathrm{GHz}$ and $7.7 \pm 0.2 \mathrm{GHz}$, respectively. This small difference in $\omega_{L}$ is within the experimental error due to the noisy low amplitude signal at negative delays, which results in a significant broadening of the FFT spectrum and a strong noise peak around zero frequency. These results also support the incomplete dephasing of the electron spin coherence within a laser pulse interval of $13.2 \mathrm{~ns}$.

We consider now the Kerr rotation signals measured on the $n$-doped CdSe/Zn(S,Se) QDs for different magnetic fields in the delay range up to $1 \mathrm{~ns}$, see Fig. 3(a). At zero magnetic field the light induced spin polarization decays with three components. Two of them have characteristic times of 30 and 300 ps. The third, longest lasting component exceeds the lifetimes of excitons and trions and can be assigned to the spin relaxation of the resident elec-
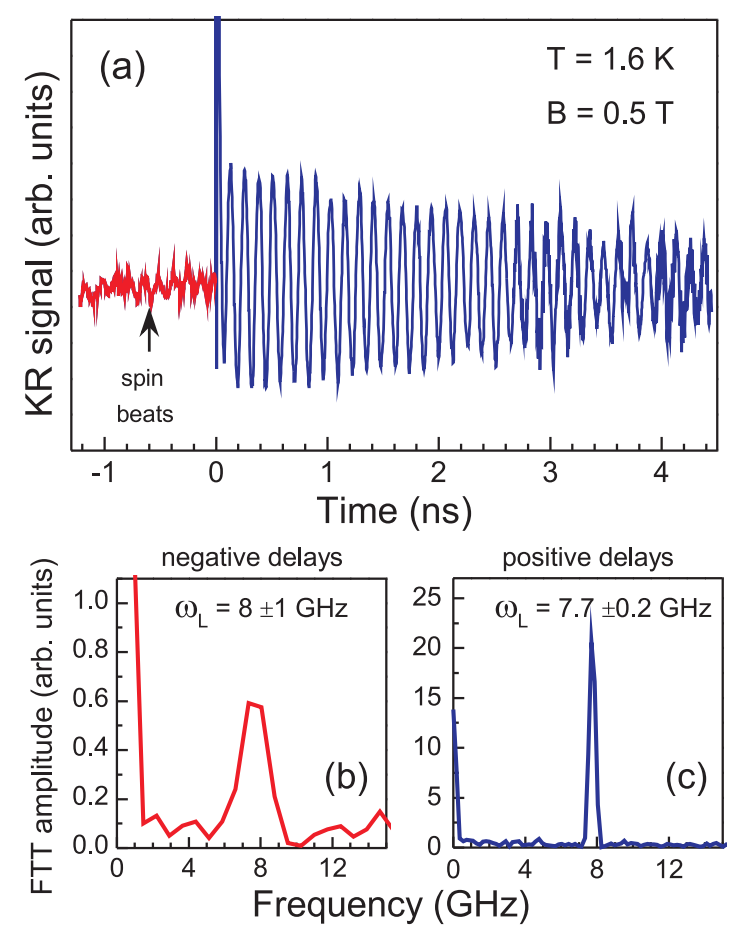

FIG. 2: (color online) (a) Time-resolved Kerr rotation trace of the $n$-doped CdSe/Zn(S,Se) QDs vs time delay between pump and probe pulses. FFT amplitudes of KR signals analyzed for negative (b) and positive (c) time delays. Pump power $60 \mathrm{~W} / \mathrm{cm}^{2}$, probe power $5 \mathrm{~W} / \mathrm{cm}^{2}$, laser photon energy $2.44 \mathrm{eV}$.

trons, while the shorter lived components correspond to relaxation processes within the trion complexes, which are limited by either spin relaxation or recombination. The signals for $B>0 \mathrm{~T}$ are dominated by long-lived oscillations with a single frequency corresponding to the resident electron Larmor frequency. Also a second nonprecessing component is present at relatively short delay times up to $250 \mathrm{ps}$. This time range coincides well with the trion and exciton lifetimes, so that the non-precessing component can be assigned to the spin dynamics of these exciton complexes. [20]

The KR signals from nominally undoped QDs are shown in Fig. 3(b). Their amplitudes are about 20 times weaker, but otherwise their appearance is qualitatively similar to that of the $n$-doped sample. The signals are dominated by long-lived electron spin beats, which can be explained by unintentional doping of a fraction of dots in the ensemble. No strong hole contribution is seen in this sample at short delays.

In the following we focus on the KR signals of the $n$ doped QD sample and analyze them for delays exceeding $200-300$ ps to study the spin coherence of resident electrons only. For this analysis we used an exponentially damped harmonic [14] to fit the KR signals and thereby obtain the spin beat frequency and the spin dephasing 


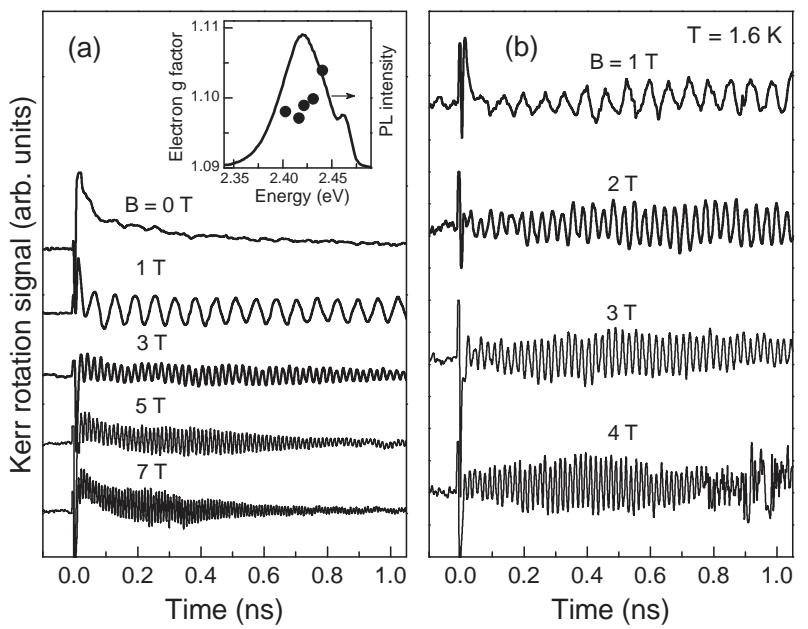

FIG. 3: KR signals in $n$-doped (a), and undoped (b) $\mathrm{CdSe} / \mathrm{Zn}(\mathrm{S}, \mathrm{Se}) \mathrm{QDs}$ at different magnetic fields. Pump power $60 \mathrm{~W} / \mathrm{cm}^{2}$, probe power $5 \mathrm{~W} / \mathrm{cm}^{2}$, laser photon energy $2.44 \mathrm{eV}$. The inset shows the electron $g$ factor dispersion across the PL band of the $n$-doped QD ensemble (circles) together with the PL spectrum (line) excited at $2.46 \mathrm{eV}$.

time:

$$
\Theta_{K R}(t)=\Theta_{K R}(0) \exp \left(-\frac{t}{T_{2}^{*}}\right) \cos \left(\omega_{L} t\right) .
$$

Here $\Theta_{K R}(0)$ is the KR angle at the moment of pump pulse arrival $t=0 \mathrm{ps}, T_{2}^{*}$ is the spin dephasing time, and $\omega_{L}$ is the Larmor spin precession frequency [21].

First, we discuss the experimental data on the inplain Landé factor $g_{e}$ of the electron. From the $\omega_{L}$ obtained through the fitting, the $g_{e}$ can be derived by: $g_{e}=\hbar \omega_{L} /\left(\mu_{B} B\right)$. The inset in Fig. 3(a) shows the dependence of $g_{e}$ on the QD emission energy. $g_{e}$ has a value of about 1.1 with a weak dispersion across the PL band originating from the $g$ factor dependence on the band gap width 25]. Our experimental technique is not sensitive to the sign of $g_{e}$, but it is known that $g_{e}$ is positive in CdSe, see Ref. 22 and references therein.

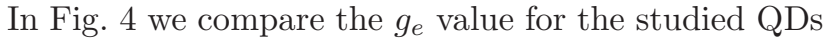
with those reported in literature. We chose a presentation form in which the $g_{e}$ is given in dependence on the energy of optical transition (corresponding to the energy gap), as this is the most straightforward way to compare the experimental results with theoretical predictions based on the Roth approach for bulk [23]. It was shown that this approach can be successfully extended also to quantum well and quantum dot heterostructures, where the quantum confinement energies have to be added to the bulk band gap energy to obtain the optical transition energies, see e.g. Refs. [9, 24, 25]. In Fig. (4 the experimental data for cubic self-organized QDs are shown by the open symbols, and for wurtzite QDs they are given by the closed symbols. Our result for $\mathrm{CdSe} / \mathrm{Zn}(\mathrm{S}, \mathrm{Se})$ dots (open circle) is in good agreement with the one for cubic CdSe/ZnSe dots (open star). [26] They are also in rea-

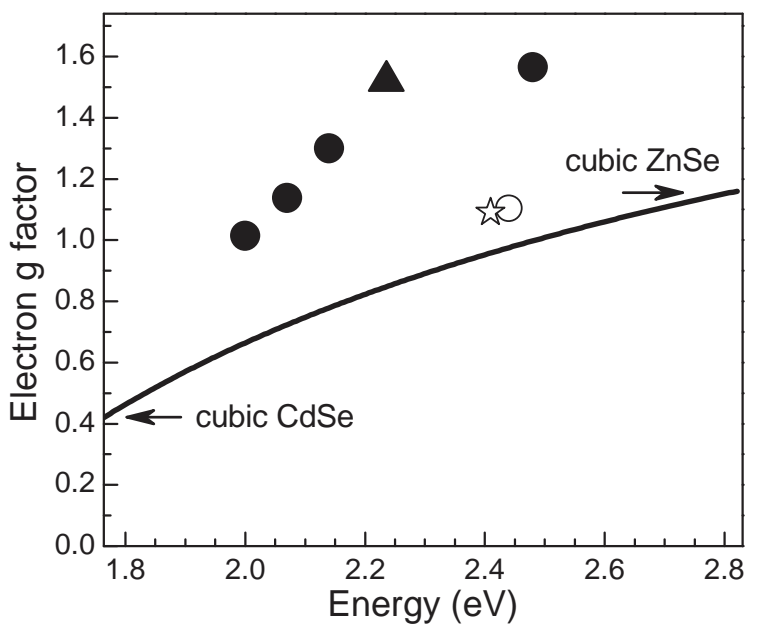

FIG. 4: Electron $g$ factor as function of optical transition energy: $n$-doped, self-assembled CdSe/Zn(S,Se) QDs (open circle), self-assembled CdSe/ZnSe QDs (open star) [26], wurtzite colloidal CdSe QDs (closed circles) [9], and wurtzite Cd(S,Se) nanocrystals in glass (closed triangle) [13]. Values for cubic bulk materials are shown by arrows: $g_{e}=0.42$ for CdSe and 1.15 for ZnSe. 22 The dispersion for cubic (Zn,Cd)Se calculated after Ref. 22] is shown by the solid line.

sonable agreement with the calculations in Ref. [22] for cubic bulk ( $\mathrm{Zn}, \mathrm{Cd}) \mathrm{Se}$, shown by the solid line. These calculations, in turn, agree well with experimental data for (Zn,Cd)Se epilayers grown on GaAs substrates. 22] However, the results for the wurtzite CdSe dots shown by the closed symbols deviate considerably from that data for cubic dots. This difference is due to differences in the band parameters for wurtzite and cubic materials, which can be also well accounted for in the Roth approach. 9]

The spin dephasing times for the resident electrons evaluated from the experimental data in Fig. 3 are presented in Fig. 5 as function of magnetic field strength. The results for $n$-doped and undoped QDs coincide well and show a strong decrease of the $T_{2}^{*}$ time from $5.6 \mathrm{~ns}$ at $B=0.25 \mathrm{~T}$ down to $300 \mathrm{ps}$ at $B=7 \mathrm{~T}$. The dephasing time is contributed by the coherence time of individual spins, $T_{2}$, and the inhomogeneous dephasing time caused by the inhomogeneity of the spin ensemble $T_{2}^{i n h}$ [14]:

$$
\frac{1}{T_{2}^{*}}=\frac{1}{T_{2}}+\frac{1}{T_{2}^{i n h}}
$$

The $T_{2}^{i n h}$ time is contributed, e.g., by the nuclear spin fluctuations (dominant at very low fields) and by the spread of the electron Larmor frequencies (dominant at high fields). The latter provides the magnetic field dependent contribution to the $T_{2}^{i n h}$ time, and therefore to the $T_{2}^{*}$ time:

$$
\frac{1}{T_{2}^{i n h}(B)}=\frac{1}{T_{2}^{i n h}(0)}+\frac{\Delta g_{e} \mu_{B} B}{\hbar} .
$$

Here $\Delta g_{e}$ is the spread of the electron $g$ factor for the optically excited spin ensemble. One can see from Fig. 5 
that the experimental data can be well described by a $1 / B$ dependence for $B>0.25 \mathrm{~T}$, as shown by the solid line assuming a $\Delta g_{e}=0.0055$. This demonstrates that the dephasing time for not too small magnetic fields is governed by the spread of the electron spin Larmor precession frequency. A similar behavior has been reported for colloidal CdSe QDs. [8, 9] Note that this spread $\Delta g_{e}=0.0055$ is less that $0.6 \%$ of the mean $g_{e}$ value.

The dephasing time $T_{2}^{*}=5.6 \mathrm{~ns}$ at $0.25 \mathrm{~T}$ is one of the longest reported so far for QDs. It is larger than the dephasing times reported for colloidal CdSe QDs of $3 \mathrm{~ns}$ at $B=0.25 \mathrm{~T}[8]$, and than $T_{2}^{*} \sim 2 \mathrm{~ns}$ at $B=0.2 \mathrm{~T}$ in (In, Ga)As/GaAs QDs. 27]

Around zero applied magnetic field the spin dephasing in quantum dots is expected to be determined by the electron interaction with the nuclear spin fluctuations at low temperatures. According to Ref. [5] the electron spin dephasing time due to this interaction can be calculated according (see Appendix for details):

$$
T_{2}^{*}=\sqrt{2} T_{\Delta}=\hbar \sqrt{\frac{3 N_{L}}{2 \sum_{j} I_{j}\left(I_{j}+1\right) \tilde{A}_{j}^{2} y_{j}}} .
$$

Here the sum runs over all types of nuclear isotopes in the dots, $N_{L}$ is the number of nuclei in the QD volume, and $I_{j}$ is the nuclear spin. $\tilde{A}_{j}$ is the hyperfine constant, which is taken for a unit cell with two nuclei, and $y_{j}$ is the probability to find the particular type of nuclear isotope (see Appendix for details). Using this equation we can evaluate the electron spin dephasing time in CdSe dots. To estimate the number of nuclei we used the geometrical volume of the dot, assumed to be of cylindrical shape (QD diameter of $4-6 \mathrm{~nm}$ and height of $1.4-2.1 \mathrm{~nm}$ ), and used hyperfine coupling constants of $-37.4 \mu \mathrm{eV}$, $39.1 \mu \mathrm{eV}$, and $33.6 \mu \mathrm{eV}$ for the stable isotopes of ${ }^{111} \mathrm{Cd}$, ${ }^{113} \mathrm{Cd}$, and ${ }^{77} \mathrm{Se}$, respectively, each having spin $1 / 2$ (see Table I and Appendix for details). This gives a $N_{L}$ in

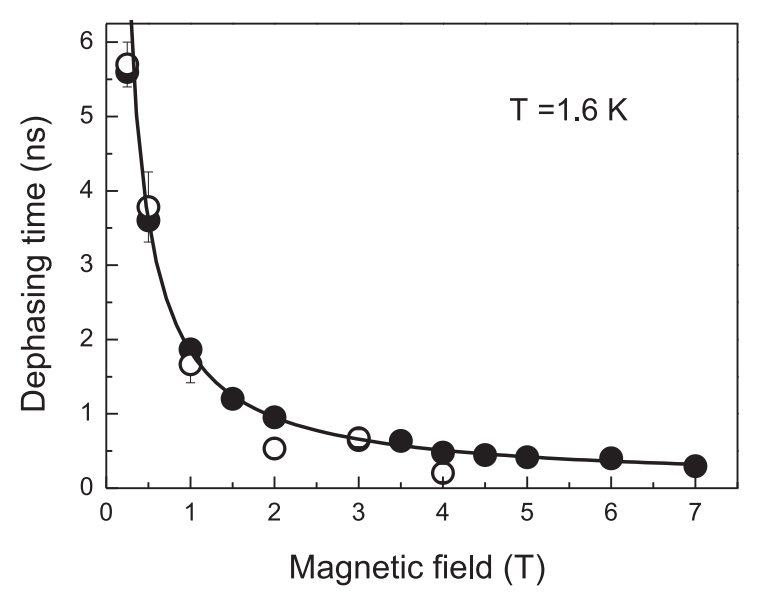

FIG. 5: Spin dephasing time $T_{2}^{*}$ vs transverse magnetic field for the $n$-doped (closed circles) and the undoped (open circles) self-assembled CdSe/Zn(S,Se) QDs. the range from 630 to 2090 nuclei and leads to a dephasing time of $1-2 \mathrm{~ns}$ depending on the QD size. For better agreement with the measured $5.6 \mathrm{~ns}$ we have to assume that either the QD volume is an order of magnitude larger, which can be achieved by assuming, for example, a height of $2.1 \mathrm{~nm}$ and a diameter of $17 \mathrm{~nm}$ or a height of $4 \mathrm{~nm}$ and a diameter of $12 \mathrm{~nm}$ (see Appendix), or the interaction with the nuclei is weaker, e.g., due to deviation of the real situation from the modeled one in the exchange-box approximation.

If the QDs would have the same size, the nucleiinduced spin dephasing would be much more efficient in GaAs and (In, Ga)As dots compared to CdSe dots, because the nuclear spins in III-V dots are considerably larger: $9 / 2$ for In and 3/2 for As and Ga compared to $1 / 2$ for $\mathrm{Cd}$ and Se. A comparison of the relevant parameters for CdSe and (In,Ga)As dots is given in Table I. In addition, $T_{2}^{\star}$ scales inversely with the number of nuclei with non-zero spin. The abundance of such isotopes in (In,Ga)As is $100 \%$ for all nuclear species, while it is only $25 \%$ for $\mathrm{Cd}$ and $7.6 \%$ for Se. This shortens the dephasing time in III-V QDs further. As a result, for dots of the same size one would expect the electron spin dephasing times to be about 10 times shorter in (In,Ga)As than in CdSe dots, see Fig. 7(a). In practice, (In,Ga)As/GaAs QDs grown by molecular-beam epitaxy have, however, considerably larger sizes than CdSe self-organized dots. This reduces the difference in the number of non-zero spin nuclei, and therefore also the difference in dephasing times.

It is important to mention though that numerical estimates based on Eq. (4) give smaller values of $T_{2}^{*}$ than experimentally observed for both CdSe and (In, Ga)As QDs. Most probably this is related to the limitations of the used approach, which is based on the "box model" assuming a constant density of the electron envelope wave function at all nuclear sites within the QD. Also possible effects of nuclear polarization, e.g. nuclear frequency focusing [28], are beyond the model consideration. Accounting for these effects would require detailed information on the QD shape and substantial computational efforts, both of which go beyond the scope of the present paper.

We examined also the temperature effect on the KR signal. Figure 6 shows how the dephasing time is changing with increasing temperature in a weak magnetic field of $B=0.25 \mathrm{~T}$. The $T_{2}^{*}$ time of the resident electrons decreases monotonically from $5.6 \mathrm{~ns}$ at $T=1.6 \mathrm{~K}\left(k_{B} T=\right.$ $0.14 \mathrm{meV})$ down to $0.8 \mathrm{~ns}$ at $T=44 \mathrm{~K}\left(k_{B} T=3.8 \mathrm{meV}\right)$.

While a strong temperature dependence of transverse electron spin relaxation times has been reported already for self-assembled QDs, e.g., (In,Ga)As/GaAs [29] and $\mathrm{InP} /(\mathrm{In}, \mathrm{Ga}) \mathrm{P}$ QDs [30], the underlying mechanisms are not trivial and are still not fully understood. They are not related to thermal escape of resident electrons, as the dot confinement potential considerably exceeds the characteristic thermal energies. Also $\Delta g_{e}$ is not expected to vary strongly in the scanned temperature range, so 


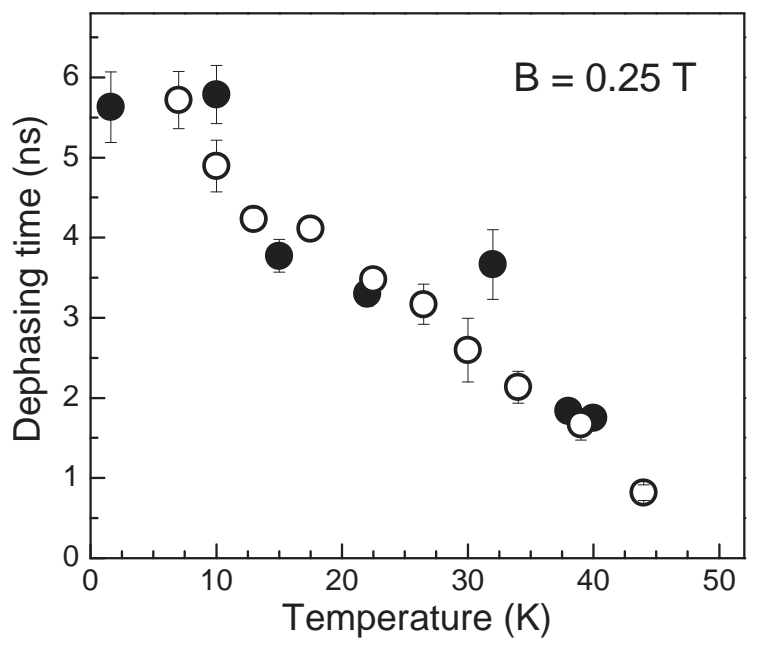

FIG. 6: Temperature dependence of the dephasing time $T_{2}^{*}$ for the $n$-doped (closed circles) and the undoped (open circles) QDs. Pump power $60 \mathrm{~W} / \mathrm{cm}^{2}$, probe power $5 \mathrm{~W} / \mathrm{cm}^{2}$, photon energy $2.43 \mathrm{eV}$.

that shortening due to related inhomogeneities can be most likely excluded.

Consequently, the shortening of the dephasing time $T_{2}^{*}$ with increasing temperature can be related to the decrease of $T_{2}$. Spin-orbit related dephasing is expected to be too weak to account for this shortening (see Ref. [29] and references therein). An effective spin relaxation mechanism providing electron spin decoherence in a quantum dot has been proposed in Ref. 31. It suggests phonon-mediated fluctuations in the electron spin precession, which are caused by the modulation of the longitudinal electron $g$ factor and the hyperfine field. Indications to that end were observed also for selfassembled (In,Ga)As/GaAs QDs 29], but more detailed studies are required to clarify the role of this mechanism for CdSe/Zn(S,Se) QDs.

\section{CONCLUSIONS}

The coherent spin dynamics of resident electrons in self-assembled CdSe/Zn(S,Se) QDs have been investigated by time-resolved photoluminescence and Kerr rotation. Long-lived electron spin coherence detectable up to $13 \mathrm{~ns}$ time delays has been found. The spin dephasing time of $5.6 \mathrm{~ns}$ at $B=0.25 \mathrm{~T}$ is one of the longest reported so far for CdSe and (In,Ga)As QDs. The magnetic field dependence of the dephasing time follows well a $1 / B$ dependence reflecting a relatively small spread of the electron $g$ factor $\Delta g_{e}=0.0055$ around the mean value of $g_{e}=1.10$ within the QD ensemble excited by the laser pulse with a spectral width of about a meV.

\section{ACKNOWLEDGMENTS}

The work was supported by the Deutsche Forschungsgemeinschaft, the EU Seventh Framework Programme (Grant No. 237252, Spin-optronics), the Russian Foundation for Basic Research (Grant No. 09-02-12406-ofi-m) and the Polish MNiSW (Grant No. N202179538). M.S. is thankful to the Foundation of Polish Science within the "START" programme. The research stay of I.A.Y. in Dortmund is supported by the Alexander von Humboldt Foundation, Bonn.
[1] Spin Physics in Semiconductors, Ed. M. I. Dyakonov (Springer-Verlag, Berlin, 2008).

[2] Semiconductor Quantum Bits, Eds. F. Henneberger and O. Benson (World Scientific, Singapore, 2008).

[3] Optical generation and control of quantum coherence in semiconductor nanostructures, Eds. G. Slavcheva and Ph. Roussignol (Springer-Verlag, Berlin, 2010).

[4] F. Meyer and B. P. Zakharchenya, Optical Orientation (North-Holland, Amsterdam, 1984).

[5] I. A. Merkulov, Al. L. Efros, and M. Rosen, Phys. Rev. B 65, 205309 (2002).

[6] A. Khaetskii, D. Loss, and L. Glazman, Phys. Rev. B 67, 195329 (2003).

[7] I. A. Akimov, D. H. Feng, and F. Henneberger, in Semiconductor Quantum Bits, Eds. F. Henneberger and O. Benson (World Scientific, Singapore,2008), Ch. 4, p. 81.

[8] J. A. Gupta, D. D. Awschalom, X. Peng, and A. P. Alivisatos, Phys. Rev. B 59, R10421 (1999).

[9] J. A. Gupta, D. D. Awschalom, Al. L. Efros, and A. V. Rodina, Phys. Rev. B 66, 125307 (2002).

[10] M. Ouyang and D. D. Awschalom, Science 301, 1074 (2003).

[11] N. P. Stern, M. Poggio, M. H. Bartl, E. L. Hu, G.
D. Stucky, and D. D. Awschalom, Phys. Rev. B 72, 161303(R) (2005).

[12] Y. Q. Li, D. W. Steuerman, J. Berezovsky, D. S. Seferos, G. C. Bazan, and D. D. Awschalom, Appl. Phys. Lett. 88, 193126 (2006).

[13] J. A. Gupta and D. D. Awschalom, Phys. Rev. B 63, 085303 (2001).

[14] D. R. Yakovlev and M. Bayer, in Spin Physics in Semiconductors, Ed. M. I. Dyakonov (Springer-Verlag, Berlin, 2008), Ch. 6, p.135.

[15] A. Shabaev, Al. L. Efros, D. Gammon, and I. A. Merkulov, Phys. Rev. B 68, 201305(R) (2003).

[16] A. Greilich, R. Oulton, E. A. Zhukov, I. A. Yugova, D. R. Yakovlev, M. Bayer, A. Shabaev, Al. L. Efros, I. A. Merkulov, V. Stavarache, D. Reuter, and A. Wieck, Phys. Rev. Lett. 96, 227401 (2006).

[17] F. Gindele, U. Woggon, W. Langbein, J. M. Hvam, K. Leonardi, D. Hommel, H. Selke, Phys. Rev. B 60, 8773 (1999).

[18] B. Patton, W. Langbein, and U. Woggon, Phys. Rev. B 68, 125316 (2003).

[19] M. Sugawara, Phys. Rev. B 51, 10743 (1995).

[20] I. A. Yugova, A. Greilich, E. A. Zhukov, D. R. Yakovlev, 
M. Bayer, D. Reuter, and A. D. Wieck, Phys. Rev. B 75, 195325 (2007).

[21] The used form $\sim \exp \left(-t / T_{2}^{*}\right) \cos \left(\omega_{L} t\right)$ to fit the time evolution of the KR amplitude assumes that the resident electrons have a Lorentzian distribution of Larmor precession frequencies, e.g., due to a spread of $g$ factor. For a Gaussian distribution the following function should be used: $\sim \exp \left(-0.5\left(t / T_{2}^{*}\right)^{2}\right) \cos \left(\omega_{L} t\right)$. However, fits of the experimental data with both functions yield approximately the same estimates for $T_{2}^{*}$.

[22] O. Z. Karimov, D. Wolverson, J. J. Davies, S. I. Stepanov, T. Ruf, S. V. Ivanov, S. V. Sorokin, C. B. O'Donnell, and K. A. Prior, Phys. Rev. B 62, 16582 (2000).

[23] L. M. Roth, B. Lax, and S. Zwerdling, Phys. Rev. 114, 90 (1959).

[24] A. A. Sirenko, T. Ruf, M. Cardona, D. R. Yakovlev, W. Ossau, A. Waag, and G. Landwehr, Phys. Rev. B 56, 2114 (1997).

[25] I. A. Yugova, A. Greilich, D. R. Yakovlev, A. A. Kiselev, M. Bayer, V. V. Petrov, Yu. K. Dolgikh, D. Reuter, and A. D. Wieck, Phys. Rev. B 75, 245302 (2007).

[26] J. Puls, M. Rabe, H.-J. Wünsche, and F.Henneberger, Phys. Rev. B 60, R16303 (1999).

[27] A. Greilich, D. R. Yakovlev, A. Shabaev, A. L. Efros, I. A. Yugova, R. Oulton, V. Stavarache, D. Reuter, A. Wieck, and M. Bayer, Science 313, 341 (2006).

[28] A. Greilich, A. Shabaev, D. R. Yakovlev, A. L. Efros, I. A. Yugova, D. Reuter, A. D. Wieck, and M. Bayer, Science 317, 1896 (2007).

[29] F. G. G. Hernandez, A. Greilich, F. Brito, M. Wiemann, D. R. Yakovlev, D. Reuter, A. D. Wieck, and M. Bayer, Phys. Rev. B 78, 041303(R) (2008).

[30] Y. Masumoto, S. Oguchi, B. Pal, and M. Ikezawa, Phys. Rev. B 74, 205332 (2006).

[31] Y. G. Semenov and K. W. Kim, Phys. Rev. Lett. 92, 026601 (2004).

[32] D. Paget, G. Lampel, B. Sapoval, and V.I. Safarov, Phys. Rev. B 15, 5780 (1977).

[33] A. Nakamura, D. Paget, C. Hermann, C. Weisbuch and G. Lampel, Solid State Commun. 30, 411 (1979).

[34] C. Testelin, F. Bernardot, B. Eble, and M. Chamarro, Phys. Rev. B 79, 195440 (2009).

[35] A. Abragam, Principles of Nuclear Magnetism (Clarendon, Oxford, 1996).

[36] American Institute of Physics Handbook, Third edition, Ch. 8b, ed. Dwight E. Gray, McGraw-Hill Book Company, New York (1972).

[37] H. Landoldt and R. Börnstein, Numerical Data and Functional Relationships in Science and Technology, Landolt-Börnstein, New Series, Vol. III.17.A (Springer, Berlin, 1982).

[38] M. Gueron, Phys. Rev. 135, A200 (1964).

[39] H. Landoldt and R. Börnstein, Semiconductors: IIVI Compounds; Semimagnetic Compounds, LandoltBörnstein, Vol. III.41.B (Springer, Berlin, 1999).

\section{Appendix A}

Here we give details of the evaluation of the nuclear spin fluctuation contribution to the dephasing time for an electron spin ensemble in singly charged QDs. Note, that for the hyperfine constants $A_{j}$ of a particular nuclear species different values can be found in literature [5, 32 34. The reason for that are differences in defining the crystal unit cell for which the $A_{j}$ are calculated. Further the isotope abundance was either included [5] or not [34] in $A_{j}$.

The Hamiltonian of hypefine Fermi contact interaction between electron and nuclear spins is: 35.

$$
\hat{H}_{h f}=\sum_{k} a_{k}\left(\hat{\boldsymbol{S}} \hat{\boldsymbol{I}}_{k}\right)
$$

where the sum goes over all nuclei, $\hat{\boldsymbol{S}}$ is the spin operator of the electron, $\hat{\boldsymbol{I}}_{k}$ is the spin operator of the $k$-th nucleus, $a_{k}=v_{0} A_{k}\left|\psi\left(\boldsymbol{R}_{k}\right)\right|^{2}, v_{0}$ is the unit cell volume, $A_{k}$ is the hyperfine constant:

$$
A_{k}=\frac{16 \pi \mu_{B} \mu_{k}\left|u_{c}\left(\boldsymbol{R}_{k}\right)\right|^{2}}{3 I_{k}}
$$

with $\mu_{k}$ and $I_{k}$ being the magnetic moment and spin of the $k$-th nucleus, the Bohr magneton $\mu_{B}$, the electron envelope wave function $\psi\left(\boldsymbol{R}_{k}\right)$ at the $k$-th nucleus, and $u_{c}\left(\boldsymbol{R}_{k}\right)$ being the electron Bloch function at the $k$ th nucleus. We write the electron wave function in the form $\Psi\left(\boldsymbol{R}_{k}\right)=v_{0} \psi\left(\boldsymbol{R}_{k}\right) u_{c}\left(\boldsymbol{R}_{k}\right)$, as in Ref. [5]. The normalization conditions are $\int_{V}\left|\psi\left(\boldsymbol{R}_{k}\right)\right|^{2} d v=1$ and $\int_{v_{0}}\left|u_{c}\left(\boldsymbol{R}_{k}\right)\right|^{2} d v=1$. With this definition $\left|u_{c}\left(\boldsymbol{R}_{k}\right)\right|^{2} \sim$ $1 / v_{0}$, and therefore one can see from Eq. (A2) that the $A_{k}$ are also $\sim 1 / v_{0}$.

To estimate the electron spin dephasing time we need to calculate the dispersion of the hyperfine field distribution $\Delta_{B}^{2}$. We assume that the fluctuating nuclear field is sum of the independent contributions of the different types of nuclei in the dots. Therefore, the dispersion $\Delta_{B}^{2}$ has to be calculated as sum of the dispersions from different contributions:

$$
\Delta_{B}^{2}=\sum_{j}\left(\Delta_{B}^{j}\right)^{2}
$$

where the sum runs over the nuclei types. Each individual contribution is:

$$
\left(\Delta_{B}^{j}\right)^{2}=\frac{2 v_{0}^{2} I_{j}\left(I_{j}+1\right)}{3\left(g_{e} \mu_{B}\right)^{2}} \sum_{j, \xi}\left(A_{j, \xi}\right)^{2}\left|\psi\left(\boldsymbol{R}_{j, \xi}\right)\right|^{4},
$$

with the sum with $\xi$ running over all nuclei of the same type $j$ in a QD. We introduce the dimensionless electron density $\eta_{j}=\left|u_{c}\left(\boldsymbol{R}_{j}\right)\right|^{2} v_{0}$ which has its maximum at the $j$-th nucleus, and also the number of nuclei in the volume of electron localization $V_{L}$ :

$$
N_{L}=\frac{n V_{L}}{v_{0}}
$$

where $V_{L}=\left(\int d v|\psi(\boldsymbol{r})|^{4}\right)^{-1}$ and $n$ is the number of nuclei in the unit cell. For the unit cell with two nuclei $(n=2): N_{L}=2 V_{L} / \tilde{v}_{0}, \tilde{A}_{j} \equiv 16 \pi \mu_{B} \mu_{j} \eta_{j} /\left(3 I_{j} \tilde{v}_{0}\right)$, 
where $\tilde{v}_{0}$ is the unit cell volume. For an unstrained cell $\tilde{v}_{0}=a_{0}^{3} / 4$, where $a_{0}$ is the lattice constant.

One can replace the sum over unit cells by an integration and evaluate the sum for all nuclei of the same type in a cell:

$$
\left(\Delta_{B}^{j}\right)^{2}=\frac{4}{3\left(g_{e} \mu_{B}\right)^{2}} \frac{1}{N_{L}} I_{j}\left(I_{j}+1\right) \tilde{A}_{j}^{2} y_{j}
$$

where $y_{j}$ is the probability to find a nucleus of type $j$. $y_{j}=x_{j} \varkappa_{j}$, where $\varkappa_{j}$ is the isotope abundance $(\varkappa \in[0,1]$ as in Ref. [32]) and $x_{j}$ is the concentration of the substitution ions in a ternary alloy, e.g., in $\operatorname{In}_{x} \mathrm{Ga}_{1-x}$ As. For binary materials $x_{j}=1$.

Thereby we obtain an electron spin dephasing time of:

$$
T_{2}^{*}=\hbar \sqrt{\frac{3 N_{L}}{2 \sum_{j} I_{j}\left(I_{j}+1\right) \tilde{A}_{j}^{2} y_{j}}} .
$$

Here the sum is over the nuclear species. The experimental value of spin dephasing time corresponds approximately to the half width at half maximum of the normal distribution [21]. Therefore the relation to the measured $T_{2}^{*}$ can be made through $T_{2}^{*}=\sqrt{2} T_{\Delta}$, where $T_{\Delta}$ is equivalent to the time introduced by Eq.(11) in Ref. [5]. The difference in coefficients is accounted for by an other choice of the unit cell with 8 atoms in Ref. [5], instead of 2 atoms used in our paper.

The values of $\eta_{j}$ for nuclei $\mathrm{Ga}, \mathrm{As}, \mathrm{In}, \mathrm{Cd}$, and Se are given in Table I. The data for $\mathrm{Ga}$, As, In, and $\mathrm{Cd}$ were taken from Refs. [32, 33, 38]. In these papers the unit cell containing two nuclei has been chosen, i.e., $v_{0}=a_{0}^{3} / 4$. To get $\eta_{j}$ we used $a_{0}(\mathrm{GaAs})=0.565 \mathrm{~nm} \mathrm{37}$, $a_{0}(\mathrm{InSb})=0.648 \mathrm{~nm}[38]$, and $a_{0}(\mathrm{CdTe})=0.648 \mathrm{~nm}$ [39]. For Se we did not find a value of $\eta_{\mathrm{Se}}$ in literature. We assume that the bonds in CdSe are covalent and take $\eta_{\text {Se }}=\eta_{\text {Cd }}$ for our estimation, as was done in Ref. 33. Values of $\tilde{A}_{j}$ calculated with unit cells with two atoms (with $a_{0}(\mathrm{GaAs})=0.565 \mathrm{~nm}$ for $\mathrm{In}, \mathrm{Ga}$, As nuclei and with $a_{0}(\mathrm{CdSe})=0.6077 \mathrm{~nm}$ for $\mathrm{Cd}$, Se nuclei) are given in Table I.

Numerical calculations give $T_{2}^{*} \approx 43.7 \sqrt{N_{L}}$ [ps] for CdSe $\left(\right.$ with $a_{0}(\mathrm{CdSe})=0.6077 \mathrm{~nm}$ [39]). The equiva- lent dependence for GaAs is $T_{2}^{*} \approx 6.63 \sqrt{N_{L}}[\mathrm{ps}]$ and for $\mathrm{In}_{x} \mathrm{Ga}_{1-x} \mathrm{As}: T_{2}^{*} \approx 6.63 \sqrt{N_{L}} / \sqrt{(1+11.61 x)}[\mathrm{ps}]$. To calculate the last expression we used the $a_{0}$ of GaAs $\left(a_{0}=0.565 \mathrm{~nm}\right)$ also for the ternary alloys, independent of $x$ which is justified because of the weak dependence of $a_{0}$ on In concentration: $a_{0}=0.565(1+0.072 x)[\mathrm{nm}]$ [37].

Figure 7(a) shows the calculated dependencies of the electron spin dephasing time $T_{2}^{*}$ on the number of nuclei $N_{L}$ in the quantum dot, for CdSe, GaAs and $\mathrm{In}_{0.2} \mathrm{Ga}_{0.8} \mathrm{As}$. Figure 7 (b) gives the dependence of $T_{2}^{*}$ on In concentration in $\operatorname{In}_{x} \mathrm{Ga}_{1-x}$ As for $N_{L}=10^{5}$.

The estimation of the number of nuclei $N_{L}$ in CdSe QDs (embedded in a ZnSe matrix grown on a (001)oriented GaAs substrate) is done as follows. We assume cylinder-shaped pancake QDs so that volume is given by QD $V_{Q D}=\pi d^{2} h / 4$, where $d$ and $h$ are diameter and
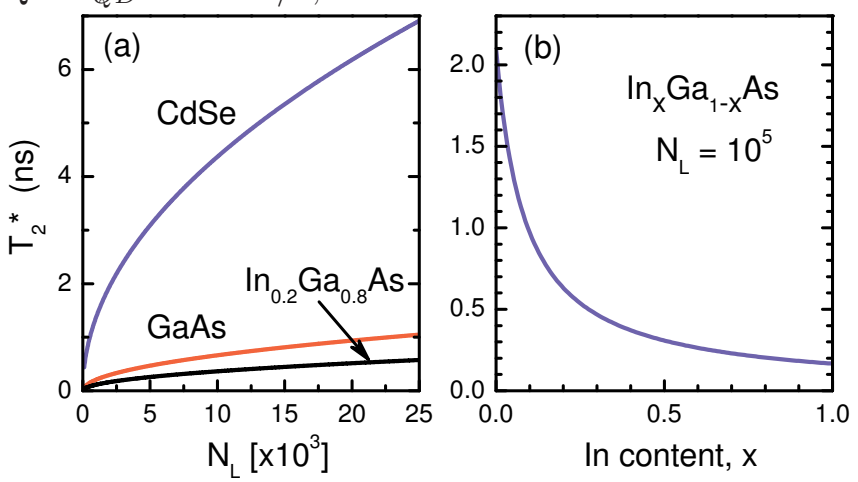

FIG. 7: (Color online) Model calculations of the nuclei fluctuation effect on the electron spin dephasing time $T_{2}^{*}$ : (a) Dependence of $T_{2}^{*}$ on the number of nuclei $N_{L}$ in the volume of electron localization for CdSe, GaAs and $\operatorname{In}_{0.2} \mathrm{Ga}_{0.8} \mathrm{As}$; (b) Dependence of $T_{2}^{*}$ on In concentration in $\operatorname{In}_{x} \mathrm{Ga}_{1-x}$ As for $N_{L}=10^{5}$.

height of the QD, respectively. We assume that the QD has almost zincblende crystal structure. Each cubic cell contains $4 \mathrm{Cd}-\mathrm{Se}$ molecules, i.e. 8 atoms. Then the volume of the cell is $v_{0}=a_{0}^{3} . \quad N_{L} \approx 8 V_{Q D} / v_{0}$. For $d \sim 4-6 \mathrm{~nm}$ and $h \sim 1.4-2.1 \mathrm{~nm}$ one obtains $N_{L} \approx 630-2090$. 
TABLE I: Parameters of nuclear isotopes used for calculating electron spin dephasing times. Only isotopes with nonzero spin are given here. All data for nuclear spins, $I_{j}$, and magnetic momenta, $\mu_{j}$, are taken from Ref. [36]

\begin{tabular}{|l|l|l|l|l|l|}
\hline Species & $I_{j}$ & $\mu_{j}$ & $\begin{array}{l}\text { Abundance } \\
\varkappa_{j}\end{array}$ & $\eta_{j}, \times 10^{3}$ & $\tilde{A}_{j}, \mu \mathrm{eV}$ \\
\hline${ }^{111} \mathrm{Cd}$ & $1 / 2$ & -0.5943 & 0.128 & $3.6^{a}$ & -37.4 \\
\hline${ }^{113} \mathrm{Cd}$ & $1 / 2$ & -0.6217 & 0.123 & $3.6^{a}$ & -39.1 \\
\hline${ }^{77} \mathrm{Se}$ & $1 / 2$ & +0.534 & 0.0758 & 3.6 & 33.6 \\
\hline${ }^{69} \mathrm{Ga}$ & $3 / 2$ & +2.016 & 0.604 & $2.61^{b}$ & 38.2 \\
\hline${ }^{71} \mathrm{Ga}$ & $3 / 2$ & +2.562 & 0.396 & $2.61^{b}$ & 48.5 \\
\hline${ }^{75} \mathrm{As}$ & $3 / 2$ & +1.439 & 1 & $4.42^{b}$ & 46 \\
\hline${ }^{113} \mathrm{In}$ & $9 / 2$ & +5.523 & 0.0428 & $6.35^{c}$ & 84.6 \\
\hline${ }^{115} \mathrm{In}$ & $9 / 2$ & +5.534 & 0.9572 & $6.35^{c}$ & 84.8 \\
\hline
\end{tabular}

${ }^{a}$ From Ref. 33.

${ }^{b}$ From Ref. 32

${ }^{c}$ From Ref. 38] 